\title{
APPLICATION OF ORTHOGONALITY RELATIONS TO SINGULAR INTEGRAL EQUATIONS
}

\author{
C.V.M. VAN DER MEE AND P.F. ZWEIFEL
}

\begin{abstract}
Singular integral equations with Cauchy type kernels are considered on a real interval. A generalization of the partial range orthogonality relations of neutron transport theory is used to generate solutions without the need of introducing the Hilbert transform, as in the standard treatment. This method has the advantage of clarifying the origin of the "endpoint conditions" in the solution of the Riemann-Hilbert problem, and, in addition, it simplifies the treatment of embedded eigenvalues.
\end{abstract}

1. Introduction. Singular integral equations of Cauchy type arise in a number of fields of physics and engineering; the general theory has been studied extensively and is described, for example, in books by Muskhelishvili [13], Gohberg and Krupnik [9] and Prößdorf [15] and a recent review paper by Estrada and Kanwal [8]. (A description of applications in transport theory has been given also, by Case [4].)

In this paper, we consider the case that the range of integration is the bounded closed interval $[-1,1]$. The simplest such equation can be written

$$
f(t)=A(t) \lambda(t)+\mathcal{P} \int_{-1}^{1} \frac{A(\nu) \eta(\nu)}{\nu-t} d \nu, \quad t \in(-1,1) .
$$

We assume that $f, \lambda$ and $\eta$ are real-valued uniformly Hölder continuous functions, although the extension to $f \in L_{p}[-1,1], 1<p<\infty$, is straightforward. The methods we are going to describe can be adapted easily to more general Cauchy type equations, such as those described in Chapter 6 of [13] or in $\S 4$ of $[\mathbf{8}]$, but the general techniques are more easily understood in the simplest case which, anyway, covers many applications of physical interest.

In addition to (1), we shall discuss the associated equation

$$
g(t)=B(t) \lambda(t)+\eta(t) \mathcal{P} \int_{-1}^{1} \frac{B(\nu)}{\nu-t} d \nu, \quad t \in(-1,1) .
$$


Note that, under the transformations

$$
A(t) \rightarrow B(t) / \eta(t),
$$

$$
f(t) \rightarrow g(t) / \eta(t),
$$

Equation (1) is replaced by $\left(1^{*}\right)$. Thus, there is no particular reason to discuss $(1)$ and $\left(1^{*}\right)$ separately unless $\eta\left(t_{0}\right)=0$ for some $t_{0} \in[-1,1]$ and $g\left(t_{0}\right) \neq 0$. A specific example of such an equation, arising in the theory of plasma oscillations, is discussed in $\S 3$.

For the time being, we restrict our attention to (1). The classical method of solution, amply discussed in the previously cited references, proceeds through the Hilbert transform

$$
N(z)=\frac{1}{2 \pi i} \int_{-1}^{1} \frac{A(\nu) \eta(\nu)}{\nu-z} d \nu
$$

and the inversion formula

$$
A(t)=\frac{1}{\eta(t)}\left[N^{+}(t)-N^{-}(t)\right]
$$

where

$$
\begin{aligned}
N^{ \pm}(t) & =\operatorname{Lim}_{e \downarrow 0} N(t \pm i \epsilon) \\
& =\frac{1}{2 \pi i} \mathcal{P} \int_{-1}^{1} \frac{A(\nu) \eta(\nu)}{\nu-t} d \nu \pm \frac{1}{2} \eta(t) A(t) .
\end{aligned}
$$

Using (3d), Equation (1) is converted to an equation for $N^{ \pm}$. Defining further the functions $\Lambda^{ \pm}$by

$$
\Lambda^{ \pm}(t)=\lambda(t) \pm \pi i \eta(t),
$$

one is led to consider the Riemann-Hilbert problem

$$
\frac{\Lambda^{+}(t)}{\Lambda^{-}(t)}=\frac{X^{+}(t)}{X^{-}(t)},
$$


where one requires $X(z)$ to be analytic on $\mathbf{C} \backslash[-1,1]$. In terms of the solution $X(z)$, the Hilbert transform solution $N(z)$ is constructed, and, ultimately, $A(t)$ is found from (3b).

One observes that the solution $X(z)$ to $(5)$ is not unique. Indeed, if $X_{0}$ is any solution, then $h(z) X_{0}(z)$ is also a solution for any analytic function $h(z)$. Let us define $X_{0}$ to be the fundamental solution

$$
\begin{gathered}
X_{0}(z)=\exp \Gamma(z), \\
\Gamma(z)=\frac{1}{2 \pi i} \int_{-1}^{1} \frac{\ln \left(\Lambda^{+}(t) / \Lambda^{-}(t)\right)}{t-z} d t .
\end{gathered}
$$

We observe that both $X_{0}(z)$ and $X_{0}(z)^{-1}=\exp \{-\Gamma(z)\}$ are analytic on $\mathbf{C} \backslash[-1,1]$ and tend to 1 as $|z| \rightarrow \infty$.

The following "endpoint conditions" have been introduced [4] as a method of removing the ambiguity on the function $X$, namely that, at the endpoints $z=1$ and $z=-1$,

$$
\operatorname{Lim}_{z \rightarrow \pm 1}( \pm 1-z) X(z)^{-1}=0
$$

and, near $z= \pm 1$,

$$
X(z)=O(1)
$$

If $X_{0}(z)$ does not obey (EC1) and (EC2) one must in general choose, for $X$,

$$
X(z)=X_{0}(z)(1-z)^{M}(1+z)^{N}
$$

for some integers $M$ and $N$ in order that it satisfies (EC1) and (EC2). (In fact, $M+N$ is identical to the "index" $\kappa$ of the singular integral equation (1) as defined in [13].) Then

(a) If $\kappa=0,(1)$ has a unique solution, which is, of course, given by the standard formula

$$
N(z)=\frac{1}{2 \pi i X_{0}(z)} \int_{-1}^{1} \eta(t) \frac{X_{0}^{+}(t)}{\Lambda^{+}(t)} \frac{f(t)}{t-z} d t
$$

with $A(t)$ recovered from $N(z)$ via (3b). 
(b) If $\kappa<0$, a solution exists only if $f(t)$ obeys the $-\kappa$ conditions

$$
\int_{-1}^{1} \eta(t) \frac{X^{+}(t)}{\Lambda^{+}(t)} f(t) t^{l} d t=0, \quad l=0,1, \ldots,-\kappa+1
$$

and this solution is unique and has the form (7).

(c) If $\kappa>0$, a solution exists, but it is not unique. To be precise,

$$
N(z)=\frac{1}{2 \pi i X(z)}\left(\int_{-1}^{1} \eta(t) \frac{X^{+}(t)}{\Lambda^{+}(t)} \frac{f(t)}{t-z} d t+P_{\kappa-1}(z)\right),
$$

where $P_{\kappa-1}$ is an arbitrary polynomial of degree $\leq \kappa-1$.

In $\S 2$ we will discuss a method for solving (1) based on a generalization of the orthogonality method introduced into transport theory by McCormick, Kusčer and Summerfield [10]. This method is a simple generalization of standard methods for computing Fourier coefficients by orthogonality and relies on the determination of a weight function and normalization integrals which allow one to write down the solution immediately. Like the Case method in transport theory [3], which was introduced because its close analogy to standard methods for solving partial differential equations made it easier to understand for physicists and engineers, our method probably will not solve any problems not already amenable to classical methods. However, Case's method in transport theory led to a much deeper understanding of the mathematical properties of transport solutions and to a flurry of activity in this field. Likewise, we think our method leads to some mathematical insight. In particular, the "endpoint conditions" which appear in a seemingly arbitrary way in [4], now enter in a most natural fashion. We also feel that our approach is pedagogically useful, in the same sense as Case's method, because of its close analogy to standard well-known techniques in mathematical physics.

One condition imposed in [13] and $[\mathbf{4}]$ is that $\Lambda^{ \pm}$not vanish anywhere on the contour of integration, in our case assumed to be the interval $[-1,1]$. One of the motivations for our work is to obtain a solution for that situation (by an appropriate limiting procedure); this is discussed in $\S 3$. In $\S 4$ an example from astrophysics is presented. 
2. The basic method. Let us write Equation (1) in the suggestive form

$$
f(t)=\int_{-1}^{1} A(\nu) \varphi_{\nu}(t) d \nu
$$

with

$$
\varphi_{\nu}(t)=\lambda(\nu) \delta(t-\nu)+\mathcal{P} \frac{\eta(\nu)}{\nu-t} .
$$

Throughout, we shall assume that $\Lambda^{ \pm}$have no zeros on $[-1,1]$. If we could find an orthogonality relation of the form

$$
\int_{-1}^{1} W(t) \varphi_{\nu}(t) \varphi_{\nu^{\prime}}(t) d t=C(\nu) \delta\left(\nu-\nu^{\prime}\right)
$$

for some weight function $W$ and some normalization coefficient $C(\nu)$, (10a) could be solved immediately as

$$
A(\nu)=\frac{1}{C(\nu)} \int_{-1}^{1} W(t) \varphi_{\nu}(t) f(t) d t
$$

which is merely an abbreviation for

$$
A(\nu)=\frac{1}{C(\nu)}\left(\mathcal{P} \int_{-1}^{1} W(t) \frac{\eta(\nu)}{\nu-t} f(t) d t+W(\nu) \lambda(\nu) f(\nu)\right)
$$

The fact that such an orthogonality relation exists and, indeed, the form of $W$ is strongly suggested by the work of Kuščer et al. previously cited [10]:

$$
W(t)=\eta(t) \frac{X^{+}(t)}{\Lambda^{+}(t)}
$$

$$
C(t)=\eta(t) X^{+}(t) \Lambda^{-}(t)=\eta(t) \frac{X^{+}(t)}{\Lambda^{+}(t)}\left[\lambda(t)^{2}+\pi^{2} \eta(t)^{2}\right]
$$


Then, writing $A_{X}(\nu)$ instead of $A(\nu)$ to indicate its dependence on $X$, (12a) reduces to

$$
A_{X}(\nu)=\frac{\lambda(\nu)}{\Lambda^{+}(\nu) \Lambda^{-}(\nu)} f(\nu)+\frac{1}{X^{+}(\nu) \Lambda^{-}(\nu)} \mathcal{P} \int_{-1}^{1} \eta(t) \frac{X^{+}(t)}{\Lambda^{+}(t)} \frac{f(t)}{\nu-t} d t
$$

Actually, (11) is not well-defined as it stands, since it involves, for example, a product of two delta functions. It is possible to define it rigorously in the context of distribution theory by introducing the Schwartz space $\mathcal{D}_{0}[(-1,1) \times(-1,1)]$, but this is not necessary. Rather, we shall take (12c) as the basic definition of orthogonality, accepting (11) as only heuristic, and prove

Proposition 1. Let $X_{0}(z)$ be the fundamental solution (6) of (5), and let $X(z)=X_{0}(z)(1-z)^{M}(1+z)^{N}$ obey (EC1) and (EC2) for some integers $M$ and $N$, where $\kappa=M+N \geq 0$. Then a solution to Equation (1) is given by $A_{X}(\nu)$ in (12).

Proof. Substitute the putative solution (12a) into Equation (10a) to obtain

$$
f(t)=\int_{-1}^{1} \frac{1}{C(\nu)} \varphi_{\nu}(t) \int_{-1}^{1} W\left(t^{\prime}\right) \varphi_{\nu}\left(t^{\prime}\right) f\left(t^{\prime}\right) d t^{\prime} d \nu .
$$

We shall show that (14) reduces to the tautology $f(t)=f(t)$. If we substitute the explicit form of $\varphi_{\nu}(t),(10 \mathrm{~b})$, and $C$ and $W,(13)$ into (14), we obtain

$$
f(t)=J_{1}+J_{2}+J_{3}+J_{4}
$$

where

$$
J_{1}=\frac{\lambda(t)^{2} f(t)}{\Lambda^{+}(t) \Lambda^{-}(t)}
$$

$$
J_{2}=\frac{\lambda(t)}{X^{+}(t) \Lambda^{-}(t)} \mathcal{P} \int_{-1}^{1} \eta\left(t^{\prime}\right) \frac{X^{+}\left(t^{\prime}\right)}{\Lambda^{+}\left(t^{\prime}\right)} f\left(t^{\prime}\right) \frac{d t^{\prime}}{t-t^{\prime}}
$$




$$
J_{3}=\mathcal{P} \int_{-1}^{1} \eta\left(t^{\prime}\right) \frac{\lambda\left(t^{\prime}\right)}{\Lambda^{+}\left(t^{\prime}\right) \Lambda^{-}\left(t^{\prime}\right)} \frac{f}{t^{\prime}} \frac{\left(t^{\prime}\right)}{-t} d t^{\prime}
$$

(15e) $J_{4}=\mathcal{P} \int_{-1}^{1} \frac{\eta(\nu)}{X^{+}(\nu) \Lambda^{-}(\nu)}\left(\mathcal{P} \int_{-1}^{1} \eta\left(t^{\prime}\right) \frac{X^{+}\left(t^{\prime}\right)}{\Lambda^{+-}\left(t^{\prime}\right)} \frac{f\left(t^{\prime}\right)}{\nu-t^{\prime}} d t^{\prime}\right) \frac{d \nu}{\nu-t}$.

In $J_{4}$ we now use the Poincaré-Bertrand formula [13] to reverse the order of the principal value integrals, obtaining

$$
J_{4}=J_{4}^{\prime}+\frac{\pi^{2} \eta(t)^{2}}{\Lambda^{+}(t) \Lambda^{-}(t)}
$$

with

$$
J_{4}^{\prime}=\mathcal{P} \int_{-1}^{1} \eta\left(t^{\prime}\right) \frac{X^{+}\left(t^{\prime}\right)}{\Lambda^{+}\left(t^{\prime}\right)} f\left(t^{\prime}\right)\left(\mathcal{P} \int_{-1}^{1} \frac{\eta(\nu)}{X^{+}(\nu) \Lambda^{-}(\nu)} \frac{d \nu}{(\nu-t)\left(\nu-t^{\prime}\right)}\right) d t^{\prime} .
$$

Combining the residual term $\pi^{2} \eta^{2} / \Lambda^{+} \Lambda^{-}$with $J_{1}$ and recalling that $\Lambda^{+} \Lambda^{-}=\lambda^{2}+\pi^{2} \eta^{2},(15 \mathrm{a})$ reduces to

$$
J_{2}+J_{3}+J_{4}^{\prime}=0
$$

which, of course, must still be verified to complete our proof.

If we now use a partial fraction decomposition in (16b) as well as the identity (which follows from (4) and (5))

$$
\frac{\eta(\nu)}{X^{+}(\nu) \Lambda^{-}(\nu)}=\frac{1}{2 \pi i}\left(\frac{1}{X^{-}(\nu)}-\frac{1}{X^{+}(\nu)}\right)
$$

we find

$$
\begin{aligned}
J_{4}^{\prime}= & \frac{1}{2 \pi i} \mathcal{P} \int_{-1}^{1} \eta\left(t^{\prime}\right) \frac{X^{+}\left(t^{\prime}\right)}{\Lambda^{+}\left(t^{\prime}\right)} f\left(t^{\prime}\right) \\
& \times\left(\mathcal{P} \int_{-1}^{1}\left(\frac{1}{X^{+}(\nu)}-\frac{1}{X^{-}(\nu)}\right)\left(\frac{1}{\nu-t}-\frac{1}{\nu-t^{\prime}}\right) d \nu\right) \frac{d t^{\prime}}{t^{\prime}-t}
\end{aligned}
$$

Equation (17) then reduces to

$$
J_{2}+J_{3}+J_{4}^{\prime}=\mathcal{P} \int_{-1}^{1} \eta\left(t^{\prime}\right) \frac{X^{+}\left(t^{\prime}\right)}{\Lambda^{+}\left(t^{\prime}\right)} f\left(t^{\prime}\right) \frac{H\left(t^{\prime}\right)-H(t)}{t^{\prime}-t} d t^{\prime}
$$


where

$$
\begin{aligned}
H(t) & =\frac{\lambda(t)}{X^{+}(t) \Lambda^{-}(t)}+\frac{1}{2 \pi i} \mathcal{P} \int_{-1}^{1} \frac{\eta(\nu)}{X^{+}(\nu) \Lambda^{-}(\nu)} \frac{d \nu}{\nu-t} \\
& =\frac{\lambda(t)}{X^{+}(t) \Lambda^{-}(t)}-\frac{1}{2 \pi i} \mathcal{P} \int_{-1}^{1}\left(\frac{1}{X^{+}(\nu)}-\frac{1}{X^{-}(\nu)}\right) \frac{d \nu}{\nu-t}
\end{aligned}
$$

If we now use (cf. (4) and (5))

$$
\frac{\lambda(t)}{X^{+}(t) \Lambda^{-}(t)}=\frac{1}{2}\left(\frac{1}{X^{+}(t)}+\frac{1}{X^{-}(t)}\right)
$$

we obtain

(20c)

$$
H(t)=\frac{1}{2}\left(\frac{1}{X^{+}(t)}+\frac{1}{X^{-}(t)}\right)-\frac{1}{2 \pi i} \mathcal{P} \int_{-1}^{1}\left(\frac{1}{X^{+}(\nu)}-\frac{1}{X^{-}(\nu)}\right) \frac{d \nu}{\nu-t}
$$

Then a sufficient condition for Proposition 1 is that $H(t)$ is a constant independent of $t$.

Now consider the integral

$$
Q(t)=\operatorname{Lim}_{R \rightarrow \infty} \frac{1}{2 \pi i} \int_{\{|z|=R\}} \frac{1}{X(z)} \frac{d z}{z-t}=\frac{1}{X(\infty)} .
$$

If the contour of integration is deformed to exclude the branch cut $[-1,1]$, then a simple application of Cauchy's theorem (noting that $X(z)^{-1}$ is analytic on $\left.\mathbf{C} \backslash[-1,1]\right)$ and the Plemelj formulas give

$$
Q(t)=H(t)
$$

modulo contributions for the endpoints \pm 1 . These contributions vanish, however, because of (EC1). Since $X(z)^{-1}=O\left(z^{-\kappa}\right)$ at infinity, we get that $H(t)$ is a constant, which proves the proposition.

Proposition 2. Let $X(z)$ be as in Proposition 1, and let $P_{\kappa}(z)$ be a polynomial of degree $\leq \kappa-1$ such that $P_{\kappa}(z) / X(z)$ vanishes as $|z| \rightarrow \infty$. Then

$$
\frac{P_{\kappa}(\nu)}{X+(\nu) \Lambda^{-}(\nu)}
$$

is a solution of the homogeneous (1). 
Remark. (EC1) is crucial to the proof of Proposition 1, but (EC2) did not enter. In fact, let us choose two nonnegative integers $n_{1}$ and $n_{2}$ and define

$$
X_{p}(z)=\frac{X(z)}{(1-z)^{n_{1}}(1+z)^{n_{2}}}, \quad n_{1}+n_{2}=p, \quad 0 \leq p \leq \kappa .
$$

Then $X_{p}(z)$ must still obey (EC1) but, for $p>0$, will not obey (EC2). Then it is easy to show, by mimicking the proof of Proposition 1, that $A_{X_{p}}(z)$ is also a solution of (1) for every $p$. That is, (1) has $\kappa$ independent solutions. It is not difficult to verify, using Proposition 2 below, that the differenee of any two such solutions is a solution of the homogeneous equation, (1) (i.e., set $f(t)=0$ ).

Proof. The proof is completely analogous to Proposition 1. That is, (23) is substituted into (1) and the steps leading to (20) are repeated. One finds that

$$
Q(t)=H(t)=\operatorname{Lim}_{R \rightarrow \infty} \frac{1}{2 \pi i} \int_{\{|z|=R\}} \frac{P_{\kappa}(z)}{X(z)} \frac{d z}{z-t}=0
$$

by the hypotheses of the present proposition, which completes the proof.

We next consider the case in which the index $\kappa$ is negative. If we go through the proof of Proposition 1, we see that (21) no longer holds, because $X(z)^{-1}$ is unbounded at infinity; there is no hope of proving that $H(t)$ is a constant. However, we can take advantage of the fact that $H(z)=$ constant is only a sufficient condition. A necessary condition [cf. (7) and (20a)] is that

$$
\mathcal{P} \int_{-1}^{1} \eta\left(t^{\prime}\right) \frac{X^{+}\left(t^{\prime}\right)}{\Lambda^{+}\left(t^{\prime}\right)} f\left(t^{\prime}\right) \frac{H\left(t^{\prime}\right)-H(t)}{t^{\prime}-t} d t^{\prime}=0 .
$$

We will illustrate this situation with an example and then prove the general result.

Suppose $X_{0}(z) \sim(1-z)$ near $z=1$ and $X_{0}(z) \sim 1$ near $z=-1$. Then $X(z)=X_{0}(z) /(1-z)$ and $\kappa=-1$. Define

$$
X_{1}(z)=X_{0}(z) \frac{z-c}{z-1}
$$


for some $c \in \mathbf{C} \backslash[-1,1]$. Now $X_{1}(z)$ also obeys (EC1), (EC2) as well as (5) and $X_{1}(z)^{-1}=O(1)$ at infinity. Since $X_{1}(z)^{-1}$ has a pole at $z=c$, we have, following the proof of Proposition 1 and using $X_{1}(z)$ instead of $X(z)$, that (21) is replaced by

$$
Q(t)=\frac{1}{X(\infty)}+\frac{c-1}{X_{0}(c)} \frac{1}{c-t}=H(t) .
$$

Then

$$
H\left(t^{\prime}\right)-H(t)=\frac{c-1}{X_{0}(c)} \frac{1}{c-t} \frac{1}{c-t^{\prime}}\left(t^{\prime}-t\right),
$$

and (25) reduces to

$$
\int_{-1}^{1} \eta\left(t^{\prime}\right) \frac{X^{+}\left(t^{\prime}\right)}{\Lambda^{+}\left(t^{\prime}\right)} f\left(t^{\prime}\right) \frac{d t^{\prime}}{t^{\prime}-c}=0
$$

i.e., the solution is given by $A_{X_{1}}(\nu)$ but subject to the constraint (26). At first sight the solution seems to depend on the choice of the arbitrary point $c$, but this is illusory. It is easy to show (using (26)) that

$$
A_{X_{1}}(\nu)=\frac{\lambda(\nu)}{\Lambda^{+}(\nu) \Lambda^{-}(\nu)} f(\nu)+\frac{1}{X^{+}(\nu) \Lambda^{-}(\nu)} \mathcal{P} \int_{-1}^{1} \eta(t) \frac{X^{+}(t)}{\Lambda^{+}(t)} f(t) \frac{d t}{\nu-t},
$$

which is manifestly independent of $c$.

Proposition 3. Let $X(z)$ be as in Proposition 1 with $\kappa<0$. Then a solution (1) is given by $A_{X}(\nu),(12)$, if and only if the $-\kappa$ constraints

$$
\mathcal{P} \int_{-1}^{1} \eta(t) \frac{X^{+}(t)}{\Lambda^{+}(t)} f(t) t^{l} d t=0, \quad l=0,1, \cdots,-\kappa+1,
$$

are satisfied.

Proof. Define $\hat{X}(z)=\prod_{l=1}^{-\kappa}\left(z-c_{l}\right) X(z)$ where the $c_{l} \in \mathbf{C} \backslash[-1,1]$ are all distinct. Note that $\hat{X}(z)$ still obeys (5). We shall proceed as in the proof of Proposition 1, i.e., we examine under what conditions the equation

$$
f(t)=\int_{-1}^{1} A_{\hat{X}}(\nu) \varphi_{\nu}(t) d \nu
$$


is a tautology. We eventually arrive at the following expression for $H(t)$ :

$$
H(t)=1+\sum_{l=1}^{-\kappa}\left(\prod_{i \neq l} \frac{1}{c_{i}-c_{l}}\right) \frac{1}{X\left(c_{l}\right)} \frac{1}{c_{l}-t}
$$

and hence

$$
H\left(t^{\prime}\right)-H(t)=\sum_{l=1}^{-\kappa}\left(\prod_{i \neq l} \frac{1}{c_{i}-c_{l}}\right) \frac{1}{X\left(c_{l}\right)} \frac{t^{\prime}-t}{\left(c_{l}-t\right)\left(c_{l}-t^{\prime}\right)} .
$$

Then the sufficient condition, (25), becomes

$$
\sum_{l=1}^{-\kappa} \frac{1}{\left(c_{l}-t\right) X\left(c_{l}\right)} \int_{-1}^{1} \eta\left(t^{\prime}\right) \frac{X^{+}\left(t^{\prime}\right)}{\Lambda^{+}\left(t^{\prime}\right)} f\left(t^{\prime}\right) \prod_{i \neq l} \frac{c_{i}-t^{\prime}}{c_{i}-c_{l}} d t^{\prime}=0
$$

which will clearly be satisfied if (27) holds. This shows that the solution is given by $A_{\hat{X}}(\nu)$. It still remains to be proved that $A_{\hat{X}}(\nu)=A_{X}(\nu)$. The first term of $A_{X}$ is independent of $X$ [cf. (12c)], so we need only consider the second term, call it $A_{2}$,

$$
A_{2, \hat{X}}(\nu)=\frac{1}{X^{+}(\nu) \Lambda^{-}(\nu)} \prod_{i=1}^{-\kappa}\left(\nu-c_{i}\right) \int_{-1}^{1} \eta(t) \frac{X^{+}(t)}{\Lambda^{+}(t)} \prod_{i=1}^{-\kappa}\left(t-c_{i}\right) f(t) \frac{d t}{\nu-t} .
$$

By repeated partial fraction decomposition, one shows

$$
\left(\prod_{i=1}^{-\kappa}\left(\nu-c_{i}\right)\right)^{-1} \frac{1}{\nu-t}=-\prod_{l=1}^{-\kappa} \prod_{j=l}^{-\kappa} \frac{1}{\nu-c_{j}} \prod_{k=1}^{l} \frac{1}{t-c_{k}}+\prod_{n=1}^{-\kappa} \frac{1}{t-c_{n}} \frac{1}{\nu-t} .
$$

Inserting this result into (28), the first terms all vanish by virtue of (27). Only the term

$$
\prod_{n=1}^{-\kappa} \frac{1}{t-c_{n}} \frac{1}{\nu-t}
$$

survives, and this inserted into (28) gives $A_{2, \hat{X}}(\nu)$. This completes the proof. $\square$ 
Let us restate our results as

THEOREM 1. Let $X_{0}(z)$ be the fundamental solution of (5) and let $X(z)=X_{0}(z)(1-z)^{M}(1+z)^{N}$ obey (EC1) and (EC2) for some integers $M$ and $N, M+N=\kappa$.

(a) If $\kappa=0$, the unique solution to (1) is given by $A_{X}(\nu)$ in (12).

(b) If $\kappa \geq 0$, the general solution to (1) is given by

$$
A_{X}(\nu)+\frac{P_{\kappa}(\nu)}{X^{+}(\nu) \Lambda^{-}(\nu)}
$$

where $A_{X}(\nu)$ is given by $(12)$ and $P_{\kappa}(\nu)$ is an arbitrary polynomial of degree $\leq \kappa-1$.

(c) If $\kappa<0$, the solution is given by $A_{X}(\nu)$ if and only if the $-\kappa+1$ constraints

$$
\mathcal{P} \int_{-1}^{1} \eta(t) \frac{X^{+}(t)}{\Lambda^{+}(t)} f(t) t^{l} d t=0, \quad l=0,1, \ldots,-\kappa+1
$$

are satisfied.

REMARK. We have now reproduced all of the classical results and, in addition, clarified the origin of the endpoint conditions (EC1) and (EC2), as was our goal.

In the event that the range of integration in (1) is $\mathbf{R}$, there are no endpoint conditions involved and, since $X_{0}(z) \rightarrow 1$ as $|z| \rightarrow \infty$, it would seem the solution always exists. However, the integral defining $X_{0},(6 \mathrm{~b})$ may diverge.

The same situation may occur if the range of integration is semiinfinite, for example, $[0, \infty)$, in which case the endpoint condition must be satisfied at 0 and the convergence of the integral in (6b) must be guaranteed at infinity. Such a situation has been encountered in some recent work involving electron transport [14]. In this case

$$
\operatorname{Lim}_{t \rightarrow \infty} \ln \frac{\Lambda^{+}(t)}{\Lambda^{-}(t)}=\pi
$$


with

$$
\ln \frac{\Lambda^{+}(0)}{\Lambda^{-}(0)}=0
$$

Since

$$
X_{0}(z) \sim z^{-\theta(0) / \pi}
$$

for $z \approx 0$, where

$$
\theta(t)=\frac{1}{2} \ln \frac{\Lambda^{+}(t)}{\Lambda^{-}(t)}
$$

(i.e., $\theta(t)=\arg \Lambda^{+}(t)$ ), the endpoint condition is apparently satisfied at $z=0$, but the integral does not exist. We renormalize $X_{0}(t)$ by

$$
X_{R}(z)=\exp \left(\frac{1}{2} \int_{0}^{\infty} \frac{\hat{\theta}(t)}{z-t} d t\right)
$$

with

$$
\hat{\theta}(t)=\theta(t)-\pi .
$$

Therefore, $X_{R}(z)$ still obeys (5), but

$$
X_{R}(z) \sim z
$$

for $z \approx 0$. The problem is reduced to the one discussed in Theorem 1 .

The same divergence of the integral defining $X_{0}(z)$, if it occurs for the case in which the range of the integral is $\mathbf{R}$, may be amenable to renormalization. If not, a solution does not exist, except, perhaps, in the special case that $\Lambda^{ \pm}(z)$ are actually the boundary values of an analytic function $\Lambda(z)$, in which case one may choose $X(z)=\Lambda(z)$.

3. A singular example. In dealing with (1) for real-valued $\lambda$ and $\eta$, the vanishing of $\Lambda^{ \pm}$on $[-1,1]$ need not cause major difficulties if one is willing to permit singular behavior of the solution $A(t)$ and does not require the vanishing of $f$. In this case, difficulties arise.

To understand this, make the transformation $(2)$, to reduce $\left(1^{*}\right)$ to (1), solve for $A$ by orthogonality and insert the result back into (1) for verification. We obtain

$$
A(\nu)=\frac{\lambda(\nu) f(\nu)}{\Lambda^{+}(\nu) \Lambda^{-}(\nu)}+\frac{\eta(\nu)}{X^{+}(\nu) \Lambda^{-}(\nu)} \mathcal{P} \int_{-1}^{1} \frac{X^{+}(t)}{\Lambda^{+}(t)} \frac{f(t)}{\nu-t} d t
$$


(30a)

$$
f(t)=\frac{\lambda(t)^{2}+\pi^{2} \eta(t)^{2}}{X^{+}(t) \Lambda^{-}(t)} f(t)+\eta(t) \mathcal{P} \int_{-1}^{1} \frac{X^{+}\left(t^{\prime}\right)}{\Lambda^{+}\left(t^{\prime}\right)} \frac{H\left(t^{\prime}\right)-H(t)}{t^{\prime}-t} d t^{\prime},
$$

$$
H(t)=\frac{\lambda(t)}{X^{+}(t) \Lambda^{-}(t)}+\mathcal{P} \int_{-1}^{1} \frac{\eta(\nu)}{X^{+}(\nu) \Lambda^{-}(\nu)} \frac{1}{\nu-t} d \nu .
$$

Equations (30) should be compared with (20) for the case in which a solution of $(1)$ rather than $\left(1^{*}\right)$ is sought.

Assume now that $\eta\left(\nu_{0}\right)=\lambda\left(\nu_{0}\right)=0$ for some $\nu_{0} \in[-1,1]$. Then $\Lambda^{ \pm}\left(\nu_{0}\right)=0$, but $f\left(\nu_{0}\right) \neq 0$. Now, if $X(\nu)$ is non-vanishing on $[-1,1]$, then the integral in (29) does not converge. On the other hand, if we choose $X\left(\nu_{0}\right)=0$ (for example, by taking $X(z)=\left(\frac{z-\nu_{0}}{z-c}\right) X_{0}(z)$ for some $c \in \mathbf{C} \backslash[-1,1])$, then the integral in (30b) does not converge. Thus the problem, as stated, has no solution.

An example of such a situation occurs in the study of plasma oscillations through the linearized Vlasov equation [1]. In this case, as is often the case in transport theory (cf. [5, Chapter 4]), it happens that the functions $\Lambda^{ \pm}(t)$ are actually the boundary values of an analytic function, $\Lambda(z)$, called the "plasma dispersion function." In such a case, it is not necessary to introduce the Riemann-Hilbert problem (5); putting it another way, one chooses the solution $X(z)=\Lambda(z)$. Also, for the Vlasov equation the interval of integration becomes $\mathbf{R}$.

In order to obtain a solution, an obvious idea is to move the zero of $\Lambda$ off the axis, to solve the resulting equation, and then take the limit as the zero returns to the axis. It turns out that this procedure yields a reasonable solution if one is careful to retain the relevant properties of $\Lambda(z)$. For the Vlasov equation, $\Lambda$ is a real function of $z$, and, further, $\Lambda(z) \rightarrow 0$ as $|z|$ tends to infinity (cf. $[5, \S 10.3]$ ). To retain both of these properties when the zero moves off the axis, we make the replacements

$$
\lambda(t) \rightarrow \lambda(t) \frac{\left(t-\nu_{0}\right)^{2}+\varepsilon^{2}}{\left(t-\nu_{0}\right)^{2}}
$$

and

$$
\eta(t) \rightarrow \eta(t) \frac{\left(t-\nu_{0}\right)^{2}+\varepsilon^{2}}{\left(t-\nu_{0}\right)^{2}}
$$


so that $\lambda$ and $\eta$ are redefined and non-vanishing (at the end of the calculation we must take the limit as $\varepsilon \downarrow 0$ ). (Note that (31) violates the assumed continuity of $\lambda$ and $\eta$; because $\Lambda^{ \pm}$are the boundary values of $\Lambda(z)$ and hence we do not need to construct the $X$-function, the continuity assumption is not needed). The plasma dispersion function then changes to

$$
\Lambda(z) \rightarrow \Lambda_{1}(z)=\Lambda(z) \frac{\left(z-\nu_{0}\right)^{2}+\varepsilon^{2}}{\left(z-\nu_{0}\right)^{2}}
$$

or

$$
\frac{1}{\Lambda_{1}(z)}=\frac{z-\nu_{0}}{\Lambda(z)} \frac{1}{2}\left(\frac{1}{z-\nu_{0}+i \varepsilon}+\frac{1}{z-\nu_{0}-i \varepsilon}\right)
$$

Returning to (30b) for $H(t)$ and replacing $X^{+}(t)$ by $\Lambda^{+}(t)$, we find, using the method followed in the proof of Proposition 3, that

$$
H(t)=\frac{\eta(t)}{2}\left(\frac{i \varepsilon}{\Lambda^{-}\left(\nu_{0}-i \varepsilon\right)} \frac{1}{\nu-i \varepsilon-t}-\frac{i \varepsilon}{\Lambda^{+}\left(\nu_{0}+i \varepsilon\right)} \frac{1}{\nu+i \varepsilon-t}\right) .
$$

There are two terms for the contributions of the poles at $\nu_{0} \pm i \varepsilon$; the one involving $X(\infty)^{-1}=\Lambda(\infty)^{-1}$ (cf. (2)) is not present, because $\eta(t)$ tends exponentially to zero at infinity. Note that $\eta(t)$ is real and analytic [11]. Then, using the Plemelj formulas,

$$
J(t)=-\eta(t) \int_{-\infty}^{\infty} W\left(t^{\prime}\right) f\left(t^{\prime}\right) \frac{H\left(t^{\prime}\right)-H(t)}{t^{\prime}-t} d t^{\prime}
$$

reduces simply to the expression

$$
\begin{aligned}
J(t)=\operatorname{Lim}_{\varepsilon \downarrow 0} & \left(\int_{-\infty}^{\infty} \frac{f\left(t^{\prime}\right)}{\nu_{0}-i \varepsilon-t^{\prime}} d t^{\prime} \frac{1}{2} \frac{i \varepsilon}{\Lambda^{-}\left(\nu_{0}-i \varepsilon\right)} \frac{1}{\nu_{0}-i \varepsilon-t}\right. \\
& \left.-\int_{-\infty}^{\infty} \frac{f\left(t^{\prime}\right)}{\nu_{0}+i \varepsilon-t^{\prime}} d t^{\prime} \frac{1}{2} \frac{i \varepsilon}{\Lambda^{+}\left(\nu_{0}+i \varepsilon\right)} \frac{1}{\nu_{0}+i \varepsilon+t}\right) \\
=-\frac{1}{2} \frac{\eta(t)}{\nu_{0}-t} & \left(\frac{1}{\left[\Lambda^{-}\right]^{\prime}\left(\nu_{0}\right)}\left(\mathcal{P} \int_{-\infty}^{\infty} \frac{f\left(t^{\prime}\right)}{\nu_{0}-t^{\prime}} d t^{\prime}-\pi i f\left(\nu_{0}\right)\right)\right. \\
& \left.+\frac{1}{\left[\Lambda^{+}\right]^{\prime}\left(\nu_{0}\right)}\left(\mathcal{P} \int_{-\infty}^{\infty} \frac{f\left(t^{\prime}\right)}{\nu_{0}-t^{\prime}} d t^{\prime}+\pi i f\left(\nu_{0}\right)\right)\right) .
\end{aligned}
$$


This term, of course, must vanish if the original singular integral equation is to have a solution. In other words, (33) represents a constraint on the function $f(t)$, similar to $(26)$.

It should be mentioned, however, that in transport theory, where singular integral equations arise as expansions of the datum $f(t)$ in terms of the (singular) eigenfunctions $\varphi_{\nu}(t)$, one takes a somewhat different point of view; solving the singular integral equation is considered to be a proof of the completeness of these eigenfunctions. The appearance of terms like those in (33) indicates that, in fact, the singular eigenfunctions are not complete; they must be augmented by discrete terms in order to obtain a complete set. Taken in this context, (33) agrees with the discrete term in the eigenfunction expansion obtained in another way [1]. It is interesting to note that, even if $f\left(\nu_{0}\right)=0$, in general the original equation has no solution unless the following constraint is satisfied:

$$
\mathcal{P} \int_{-\infty}^{\infty} \frac{f(t)}{\nu_{0}-t} d t=0
$$

Further, if we think of the two "eigenvalues" $\nu_{0} \pm i \varepsilon$ moving towards the axis as some parameter, e.g., the electron density, is changed, they coalesce as a single root, not a double root. This agrees with calculations presented by Crawford and Hislop [7] who used an entirely different approach.

4. An example from astrophysics. In the atmospheres of the outer planets magnetic fields cause ice crystals to be oriented in a preferential direction. For ice crystals which can be treated as thin infinite cylinders the equation of transfer of polarized radiation is decomposed into scalar equations of the form [12]

$$
\lambda(\nu) A(\nu)-\mathcal{P} \int_{0}^{1} \frac{t \Psi_{0}(t) A(t)}{(t-\nu) \sqrt{1-t^{2}}} d t=\frac{1}{\nu},
$$

where

$$
\lambda(\nu)=1+\nu \mathcal{P} \int_{-1}^{1} \frac{\Psi_{0}(t)}{(t-\nu) \sqrt{1-t^{2}}} d t
$$

and $\Psi_{0}(t)$ is a polynomial in $t^{2}$, the so-called characteristic function, which is strictly positive on $[-1,1]$. Thus $\lambda(t) \neq 0$ for $t \in[0,1]$. The 
solution $A(\nu)=H(\nu) / \nu$, where $H(\nu)$ is a variant of Chandrasekhar's celebrated H-equation [6]. It should be noted that, although (34) does not formally satisfy the general assumptions of $\S 2$, the transformation $A(\nu) \rightarrow A^{\prime}(\nu)=A(\nu) /\left(1-\nu^{2}\right)^{1 / 2}$ converts it to an equation which does satisfy these assumptions.

We seek a positive solution of (34) using the methods of $\S 2$. We have

$$
\eta(\nu)=-\frac{\nu \Psi_{0}(\nu)}{\sqrt{1-\nu^{2}}}
$$

and

$$
\Lambda^{ \pm}(\nu)=\lambda(\nu) \mp \frac{i \pi \nu \Psi_{0}(\nu)}{\sqrt{1-\nu^{2}}} .
$$

The function $X_{0}(z)$ is given by (6) with

$$
\Gamma(z)=\frac{1}{\pi} \int_{0}^{1} \frac{\arg \Lambda^{+}(t)}{t-z} d t,
$$

where

$$
\theta(t)=\arg \Lambda^{+}(t)=-\arctan \frac{\pi t \Psi_{0}(t)}{\lambda(t) \sqrt{1-t^{2}}}
$$

Thus the argument runs monotonically from 0 to $-\pi / 2$. Hence,

$$
\begin{gathered}
X_{0}(z) \sim z^{\theta(0) / \pi} \sim 1 \quad \text { near } z=0, \\
X_{0}(z) \sim(1-z)^{\theta(1) / \pi} \sim(1-z)^{-1 / 2} \text { near } z=1 .
\end{gathered}
$$

Thus $X_{0}(z)$ obeys (EC1) but not (EC2). Then

$$
X_{1}(z)=(1-z) X_{0}(z)
$$

obeys both (EC1) and (EC2), so that the index $\kappa=1$. By virtue of Theorem 1 the general solution is given by

$$
\begin{aligned}
A(t)= & \frac{\lambda(t)}{\Lambda^{+}(t) \Lambda^{-}(t)} \frac{1}{t} \\
& +\frac{1}{X_{1}^{+}(t) \Lambda^{-}(t)} \mathcal{P} \int_{0}^{1} \eta(\nu) \frac{X_{1}^{+}(\nu)}{\Lambda^{+}(\nu)} \frac{d \nu}{\nu(t-\nu)}+\frac{c}{X_{1}^{+}(t) \Lambda^{-}(t)},
\end{aligned}
$$


where $c$ is an arbitrary constant. The situation where one arbitrary constant appears in the solution of the linear $H$-equation is typical of linear transport theory. This solution will satisfy the nonlinear $H$ equation $((27)$ of $[\mathbf{1 1}])$ for only two values of $c$, only one of which leads to a positive solution [2].

Acknowledgements. The authors wish to thank Kenneth Case, William Greenberg, Joseph Ball and Israel Gohberg for stimulating discussions. The research described here was supported by the U.S. Department of Energy Grant DE-FG-05-87ER25033 and National Science Foundation Grants DMS 8701050 and DMS 8823102. One of the authors (PFZ) would like to thank l'Istituto di Matematica dell' Università di Catania and Professor Marcello Anile for their hospitality during a visit in April 1987 when a part of the work described here was carried out.

\section{REFERENCES}

1. M.D. Arthur, W. Greenberg and P.F. Zweifel, Transverse plasma oscillations, Phys. Fluids 22 (1979), 1465.

2. I.W. Busbridge, The Mathematics of Radiative Transfer, Cambridge Univ. Press, Cambridge, 1960.

3. K.M. Case, Ann. Phys (N.Y.) 9, 1 (1960).

4. - "Methods for solving linear transport problems," In: E. Inönü, P.F. Zweifel (Eds.), Developments in Transport Theory, Academic Press, New York, 1967.

5. — and P.F. Zweifel, Linear Transport Theory, Addison-Wesley, Reading, Mass., 1967.

6. S. Chandrasekhar, Radiative Transfer, Oxford Univ. Press, London and New York, 1950; also Dover Publ., New York, 1960.

7. J.D. Crawford and P.D. Hislop, Application of the Method of Spectral Deformation to the Vlasov-Poisson System, VCSD Preprint, 1987.

8. R. Estrada, R.P. Kanwal, SIAM Review 29 (1987), 263-290.

9. I.C. Gohberg and N. Krupnik, Einführung in die Theorie der eindimensionalen singulären Integraloperatoren, Birkhäuser, Basel, 1979.

10. I. Kuščer, N.J. McCormick and G.C. Summerfield, Orthogonality of Case's eigenfunctions in One-Speed transport theory, Ann. Phys. (N.Y.) 30 (1964), 411.

11. L. Landau, On the vibrations of the electron plasma, J. Phys. U.S.S.R. 10 (1946), 25. 
12. M.I. Mishchenko and E.G. Yanovitskij, Transfer of polarized radiation in a medium consisting of completely oriented strongly elongated particles, Kinematics and Physics of Celestial Bodies 4 (1988), 19-29 [Russian].

13. N.I. Muskhelishvili, Singular Integral Equations, Noordhoff, Groningen, 1953.

14. S.L. Paveri-Fontana and P.F. Zweifel, in preparation.

15. S. Prößdorf, Some Classes of Singular Equations, North-Holland, Amsterdam, 1978.

Center for Transport Theory and Mathematical Physics, Virginia Polytechnic Institute and State University, Blacksburg, VA 24061 
